\title{
O EMPREGO DATERAPIA LASER DE BAIXA INTENSIDADE NO TRATAMENTO DA HERPES-ZÓSTER E NEURALGIA PÓS-HERPÉTICA COM ACOMETIMENTO OROFACIAL
}

\author{
Davidson Ribeiro Costa ${ }^{1}$ \\ David Ribeiro Costa ${ }^{2}$ \\ Diego Rodrigues Pessoa ${ }^{3}$ \\ Carolina Alves Delpasso ${ }^{4}$ \\ Renata Amadei Nicolau ${ }^{5}$
}

Resumo: O herpes-zóster facial é definido como uma doença infecciosa, que durante seu desenvolvimento apresenta lesões dolorosas na região orofacial. A Neuralgia pós-herpética é uma complicação da $\mathrm{HZ}$ facial que é caracteriza pela sintomatologia dolorosa. A terapia com laser de baixa intensidade (TLBI) tem sido amplamente divulgada nos meios científicos, devido o seu efeito positivo na redução da dor em uma série de condições. Nesse contexto, o presente estudo objetivou analisar os principais efeitos da TLBI, com base na literatura, de forma a apresentar uma terapia alternativa, que pode ser empregada no tratamento da $\mathrm{HZ}$ facial e NPHT, salientando as doses e os possíveis locais para a irradiação em cada fase. A TLBI é uma terapia não invasiva, que não apresenta efeitos adversos, quando empregada de maneira correta. Essa terapia apresenta efeitos anti-álgico, anti-inflamatório, de reparo tecidual e neuronal, o que justifica a sua utilização no tratamento da HZ facial e NPHT.

Palavras-chave: Herpes-zóster orofacial; Herpes-zóster facial; Neuralgia pós-herpética; Neuralgia póshérpetica do nervo trigêmeo; Terapia laser de baixa intensidade.

\footnotetext{
${ }^{1}$ Centro de Laserterapia e Fotobiologia (CELAFO),Instituto de Pesquisa e Desenvolvimento(IP\&D)/ Universidade do Vale do Paraíba- UNIVAP, Brasil. E-mail: dnribcosta@hotmail.com.

2 Centro de Laserterapia e Fotobiologia (CELAFO),Instituto de Pesquisa e Desenvolvimento(IP\&D)/ Universidade do Vale do Paraíba- UNIVAP, Brasil. E-mail: davidrcosta@yahoo.com.br.

${ }^{3}$ Centro de Laserterapia e Fotobiologia (CELAFO),Instituto de Pesquisa e Desenvolvimento(IP\&D)/ Universidade do Vale do Paraíba- UNIVAP, Brasil. E-mail: fisio.diegorodrigues@gmail.com.

${ }^{4}$ Centro de Laserterapia e Fotobiologia (CELAFO),Instituto de Pesquisa e Desenvolvimento(IP\&D)/ Universidade do Vale do Paraíba- UNIVAP, Brasil. E-mail: delpassoc@gmail.com.

${ }^{5}$ Centro de Laserterapia e Fotobiologia (CELAFO),Instituto de Pesquisa e Desenvolvimento(IP\&D)/ Universidade do Vale do Paraíba- UNIVAP, Brasil. E-mail: rani@univap.br.
} 